\title{
Different Strategies for Power Quality Improvement in Off-Grid Renewable Energy Based Power System
}

\author{
Rajbir Sood \\ M.Tech Scholar, Elect. Engg. Deptt. \\ BUEST, Baddi, India
}

\author{
Geena Kalpesh \\ Professor \\ BUEST, Baddi, India
}

\begin{abstract}
The increasing trend in integrating intermittent renewable energy sources into off-grid power system presents major challenges from the viewpoints of reliable operation and control. In this paper, the major problems and challenges in off-grid power system control are discussed, and a review of control strategies and trends is presented. A general overview of the main control is also included. The paper classifies power quality improvement strategies into three levels: primary, secondary, and tertiary, where primary and secondary levels are associated with the operation of the off-grid power system itself, and tertiary level pertains to the coordinated operation of the power system. Each control level is discussed in detail in view of the relevant existing technical literature.
\end{abstract}

KEYWORD: Off-grid power system, load frequency control, power electronics converters, energy storage system.

\section{INTRODUCTION}

With the expansion of the electrical power grid, conventional power system has become increasingly vulnerable to cope with the reliability requirements and the diverse demand of power users. Moreover, off-grid power system using renewable energy (RE) sources has advantages of pollution reduction, highenergy utilization rate, flexible installation location, and low-power transmission losses. Off-grid power system using renewable energy sources units also present a higher degree of controllability and operability compared to the conventional generators [1], which will allow this type of power system to play a major and critical role in maintaining the stability of electrical networks [2]-[4]. So RE based off-grid power system will gradually be a strong and effective support for the main power grid and potentially one of the future trends of power system.
The distributed generation (DG) units of a small offgrid power system can be classified into grid forming (voltage-controlled) and grid-following (current controlled) DG units. In grid-connected mode, the units are often controlled as grid-following. The most adopted control strategies for grid-following inverters are discussed in [4]. In islanding mode, the electronic converter interfaces between the loads and the microsource act as voltage sources, which are responsible for the power sharing according to their ratings and availability of power from their corresponding energy sources or prime movers [5].

In order to successfully integrate renewable Distributed Energy Resources (DER), many technical challenges must yet be overcome to ensure that the present levels of reliability are not significantly affected, and the potential benefits of distributed generation are fully harnessed. In this sense, the main issues include [6]:

- Schedule and dispatch of units under supply and demand uncertainty, and determination of appropriate levels of reserves.

- Reliable and economical operation of RE bases off-grid power system with high penetration levels of intermittent generation in stand-alone mode of operation.

- Design of appropriate control strategy schemes to allow customers to react to the grid's needs.

- Development of new voltage and frequency control techniques to account for the increase in power-electronics-interfaced distributed generation.

This concept is a quite appealing alternative for overcoming the challenges of integrating DER units, including renewable energy sources, into power systems. However, in order to allow seamless deployment of micro-grids, several issues still remain 


\section{www.ijtsrd.com}

unsolved. Currently, effort is being put into the design of special protection schemes and control systems that ensure reliable, secure and economical operation of micro-grids in either grid-connected or stand-alone mode. This paper presents a general overview of the existing technologies and remaining challenges in micro-grid control.

\section{Off-Grid Power System using RE Sources}

The concept of small off-grid power system using RE sources was first introduced in the technical literature in [7] as a solution for the reliable integration of DERs, including Energy Storage Systems (ESSs) and controllable loads. Such micro-grid would be perceived by the main grid as a single element responding to appropriate control signals. Although a detailed definition of micro-grids is still under discussion in technical forums, a micro-grid can be described as a cluster of loads, Distributed Generation (DG) units and ESSs operated in coordination to reliably supply electricity, connected to the host power system at the distribution level at a single point of connection, the Point of Common Coupling (PCC). The adoption of micro-grids as the paradigm for the massive integration of distributed generation will allow technical problems to be solved in a decentralized fashion, reducing the need for an extremely ramified and complex central coordination and facilitating the realization of the Smart Grid.

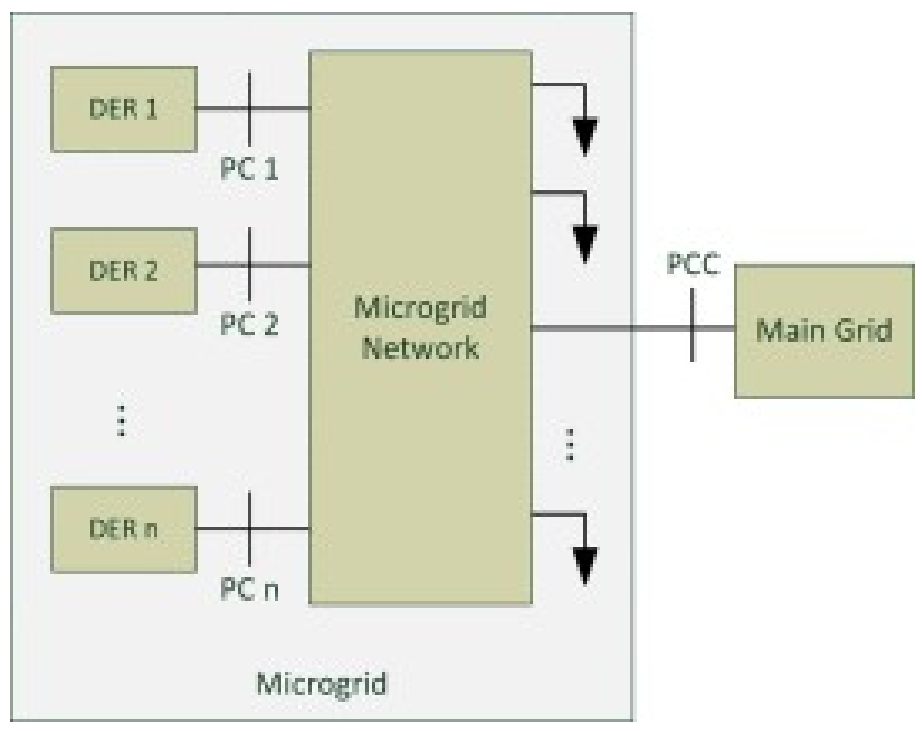

Fig. 1. Schematic diagram of a generic multiple-DER micro-grid

In general, a micro-grid can have any arbitrary configuration, as illustrated in Fig. 1; however, some entities, such as the Consortium for Electric Reliability Technology Solutions (CERTS), promote a configuration in which loads are connected to the feeders with existing generation [8]. In some cases, where a strong coupling between the operation of different energy carrier systems (heating, hot water, etc.) exists, micro-grids can integrate and operate all these energy carriers in coordination.

\section{Requirements for Control and Protection}

Micro-grids and integration of DER units in general, introduce a number of operational challenges that need to be addressed in the design of control and protection systems in order to ensure that the present levels of reliability are not significantly affected and the potential benefits of DG are fully harnessed. Some of these challenges arise from invalid assumptions typically applied to conventional distribution systems, while others are the result of stability issues formerly observed only at a transmission system level.

The most relevant challenges in micro-grid protection and control include:

Bidirectional power flows: While distribution feeders were initially designed for unidirectional power flow, integration of DG units at low voltage levels can cause reverse power flows and lead to complications in protection coordination, undesirable power flow patterns, fault current distribution, and voltage control.

Stability issues: Local oscillations may emerge from the interaction of the control systems of DG units, requiring a thorough small-disturbance stability analysis. Moreover, transient stability analyses are required to ensure seamless transition between the grid-connected and stand-alone modes of operation in a micro-grid.

> Modeling: Prevalence of three-phase balanced conditions, primarily inductive transmission lines, and constant-power loads are typically valid assumptions when modeling conventional systems at a transmission level; however, these do not necessarily hold valid for micro-grids, and consequently models need to be revised.

Low inertia: Unlike bulk power systems where high number of synchronous generators ensures a relatively large inertia, micro-grids might show a low-inertia characteristic, especially if there is a significant share of power electronic-interfaced DG units. Although such an interface can enhance the system dynamic performance, the low inertia in the system can lead to severe frequency 
deviations in stand-alone operation if a proper control mechanism is not implemented.

Uncertainty: The economical and reliable operation of micro-grids requires a certain level of coordination among different DERs. This coordination becomes more challenging in isolated micro-grids, where the critical demandsupply balance and typically higher component failure rates require solving a strongly coupled problem over an extended horizon, taking into account the uncertainty of parameters such as load profile and weather forecast. This uncertainty is higher than those in bulk power systems, due to the reduced number of loads and highly correlated variations of available energy resources (limited averaging effect).

The control system must be able to ensure the reliable and economical operation of the micro-grid, while overcoming the aforementioned challenges. In particular, desirable features of the control system include:

Output control: Output voltages and currents of the various (DER) units must track their reference values and ensure oscillations are properly damped. Power balance: DER units in the micro-grid must be able to accommodate sudden active power imbalances, either excess or shortage, keeping frequency and voltage deviations within acceptable ranges.

DSM: Where applicable, proper DSM mechanisms must be designed in order to incorporate the ability to control a portion of the load. Additionally, for the electrification of remote communities with abundant local renewable resources, the active participation of the local community may be beneficial in order to design cost-effective DSM strategies that enhance load-frequency control [8].

D Economic dispatch: An appropriate dispatch of DER units participating in the operation of a micro-grid can significantly reduce the operating costs, or increase the profit. Reliability considerations must also be taken into account in the dispatch of units, especially in stand-alone operation.

Transition between modes of operation: A desirable feature of micro-grids is the ability to work in both grid-connected and stand-alone modes of operation, including a smooth transition between them. Different control strategies might be defined for each mode of operation and, therefore, a high-speed islanding detection algorithm is very important in order to adjust the control strategy accordingly.

In the micro-grid environment, characterized by having frequent and multiple changes in topology, robustness of controllers are desired traits. Availability of measurements, communication, and high-speed computational facilities are additional challenges for all the above requirements; for this reason, an attempt should be made in order to reduce the need for high-speed communications and computation in critical tasks. The adoption of a hierarchical control structure is quite appealing given the different time constants involved, including fast dynamics in the output controls and slower dynamics in the economic dispatch. Complexity and sophistication of the solutions for the control requirements of the micro-grid will be very much dependent on whether it is designed to primarily operate in stand-alone or grid-connected mode. While in grid-connected mode of operation emphasis is put on the interaction with the main grid, reliability issues are more significant in off-grid mode of operation. A description of controlled variables used in micro-grid control and different types of DER units is presented next.

\section{A. Control Variables}

The main variables used to control the operation of a micro-grid are voltage, frequency, and active and reactive power. In the grid-connected mode of operation, the frequency of the micro-grid and the voltage at the PCC are dominantly determined by the host grid. The main role of the micro-grid control in this case is to accommodate the active and reactive power generated by the DER units, and the load demand. Reactive power injection by a DER unit can be used for power factor correction, reactive power supply, or voltage control at the corresponding Point of Connection (PC). In this mode, the host utility may not allow regulation or control of the voltage by DER units in proximity of the PCC (determined by the electrical distance and Short Circuit MVA of the grid) to avoid interaction with the same functionality provided by the grid [9]. In stand-alone mode of operation, the micro-grid operates as an independent entity. This mode of operation is significantly more challenging than the grid connected mode, because the critical demand-supply equilibrium requires the 
implementation of accurate load sharing mechanisms to balance sudden active power mismatches. Voltages and frequency of the micro-grid are no longer supported by a host grid, and thus they must be controlled by different DER units. Power balance is ensured either directly by local controllers utilizing local measurements, or by a central controller that communicates appropriate set points to local controllers of different DER units and controllable loads.

\section{B. Different types of DER Units}

The DER units present in a particular micro-grid are very problem-specific and depend on a variety of factors, including whether the micro-grid is designed to operate in grid-connected or stand-alone mode, the different generation technologies deployed, and the topology of the system [35]. In general, the components that can be found in a purely-electrical micro-grid are illustrated in Fig. 2.

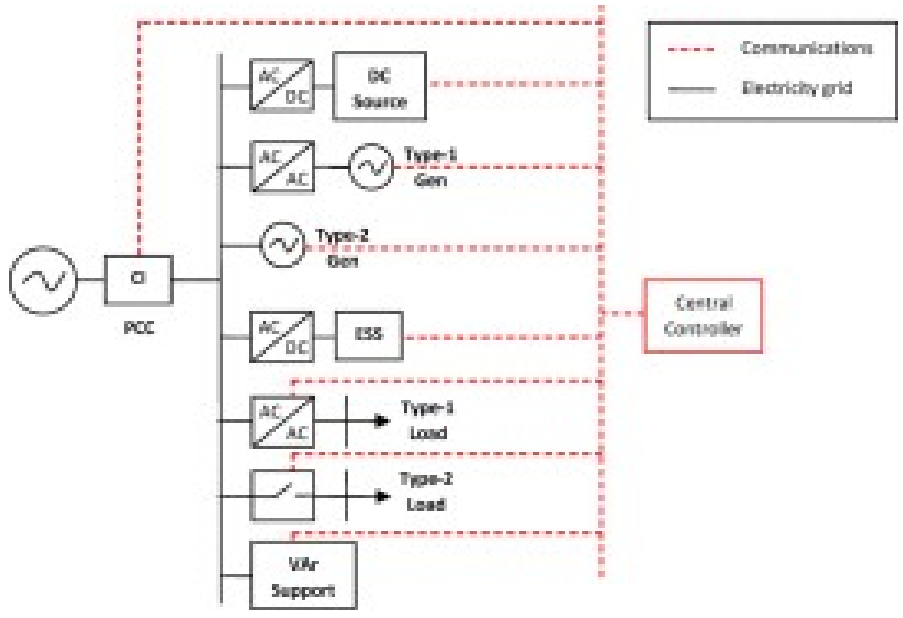

Fig. 2. Micro-grid general components

Micro-grids are characterized by a single point of connection with the host grid. The Connection Interface $(\mathrm{CI})$ at the PCC can be realized using electromechanical circuit breakers, solid state switches or even back-to-back converters. The connection of DCtype energy sources such as PV panels, fuel cells and energy with different energy and power ratings and efficiencies and in different applications (e.g., diurnal renewable resources levelizing, reserve augmentation, voltage support, and reliability enhancement) [10][14]: Battery Energy Storage System (BESS), Compressed Air Energy Storage (CAES) systems, flywheels, thermal energy storage, pumped hydro, Superconducting Magnetic Energy Storage (SMES) and vehicle-to-grid (V2G) technologies [15].

\section{Energy Storage System}

The Integrated storage can decrease losses and increase reliability. Energy storage enables large-scale integration of intermittent renewable energy sources [16]. The benefits of storage in the latter application are of particular interest, because while renewable energy resources are a pillar of the micro-grids, without storage, their generation cannot improve the system reliability and has to be duplicated by other means of generation. A storage unit can provide a functionality similar to that of the inertia of a synchronous generator by absorbing temporary mismatches between power generation and demand, especially in a low inertia power electronic-based micro-grid. Therefore, system stabilization can be improved by providing voltage/frequency control in a droop-based scheme.

Despite its benefits, energy storage has not been fully utilized. Among the limiting factors is, besides the cost, the lack of appropriate control and management strategies. Future research is needed to investigate and develop control methodologies for the following different energy storage technologies, with different energy and power ratings and efficiencies and in different applications (e.g., diurnal renewable resources levelizing, reserve augmentation, voltage support, and reliability enhancement) : Battery Energy Storage System (BESS), Compressed Air Energy Storage (CAES) systems, flywheels, thermal energy storage, pumped hydro, Superconducting Magnetic Energy Storage (SMES) and vehicle-to-grid (V2G) technologies.

\section{Load Frequency control in Small Off-grid Power System}

With regard to the architecture of a power system's control two very distinctive opposite approaches can be identified: centralized and decentralized. A fully centralized control relies on the data gathered in a dedicated central controller that performs the required calculations and determines the control actions for all the units at a single point, requiring extensive communication between the central controller and controlled units. On the other hand, in a fully decentralized control each unit is controlled by its local controller, which only receives local information and is neither fully aware of system-wide variables nor other controllers' actions [18].

Interconnected power systems usually cover extended geographic areas, making the implementation of a 


\section{www.ijtsrd.com}

fully centralized approach infeasible due to the extensive communication and computation needs. At the same time, a fully decentralized approach is also not possible due to the strong coupling between the operations of various units in the system, requiring a minimum level of coordination that cannot be achieved by using only local variables. A compromise between fully centralized and fully decentralized control schemes can be achieved by means of a hierarchical control scheme consisting of three control levels: primary, secondary, and tertiary. These control levels differ in their (i) speed of response and the time frame in which they operate, and (ii) infrastructure requirements (e.g., communication requirements). Although micro-grids are not necessarily as geographically expansive as conventional power systems, they can benefit from this control hierarchy.

The rest of this section presents an overview of different control levels from the perspective of microgrids. Subsequent sections present the state of the art in control methods pertaining to different levels.

\section{A. Primary Control}

Primary control, also known as local control or internal control is the first level in the control hierarchy, featuring the fastest response. This control is based exclusively on local measurement and requires no communication. Given their speed requirement and reliance on local measurements, islanding detection output control and power sharing (and balance) control are included in this category. In synchronous generators, output control and power sharing is performed by the voltage regulator, governor, and the inertia of the machine itself. Voltage-Source Inverters (VSIs) used as interface for DC sources, or as part of back-to-back converters, require a specially designed control to simulate the inertia characteristic of synchronous generators and provide appropriate frequency regulation. For this purpose, VSI controllers are composed of two stages: DG power sharing controller and inverter output controller. Power sharing controllers are responsible for the adequate share of active and reactive power mismatches in the micro-grid, whereas inverter output controllers should control and regulate the output voltages and currents. Inverter output control typically consists of an outer loop for voltage control and an inner loop for current regulation. Power sharing is performed without need for communication by using active power-frequency and reactive power-voltage droop controllers that emulate the droop characteristics of synchronous generators.

\section{B. Secondary Control}

Secondary control, also referred to as the micro-grid Energy Management System (EMS), is responsible for the reliable, secure and economical operation of micro-grids in either grid-connected or stand-alone mode. This task becomes particularly challenging in isolated micro-grids with the presence of highlyvariable energy sources, where the update rate of the unit dispatch command should be high enough to follow the sudden changes of load and non-dispatch able generators. The objective of the EMS consists of finding the optimal (or near optimal) Unit Commitment (UC) and dispatch of the available DER units, so that certain selected objectives are achieved. Permanent voltage and frequency deviations produced by the action of the primary control are also restored by the secondary control. In order to determine the dispatch and UC of the micro-grid, three main options are identified: real-time optimization, expert systems, and decentralized hierarchical control.

For the EMS architecture, two main approaches can be identified: centralized and decentralized architectures. Secondary control is the highest hierarchical level in micro-grids operating in standalone mode, and operates on a slower time frame as compared to the primary control in order to (i) decouple secondary control from primary control, (ii) reduce the communication bandwidth by using sampled measurements of the micro-grid variables, and (iii) allow enough time to perform complex calculations. The typically limited geographical span of micro-grids facilitates communication through affordable and simple standard protocols, which needs to be of low bandwidth and only for slowly changing parameters, such as set points for real and reactive power. According to the Galvin electricity initiative [16], a central controller is required to ensure that the power system operation is as seamless as possible during major disturbances such as transition from grid-connected mode to islanded mode. In the proposed approach, the master controller is responsible for economic optimization of the microgrid whenever possible (i.e., in non-emergency mode of operation and when connected to the main grid), as well as maintaining reliable, secure, and safe operation of the grid. In [17], optimal operation is sought through the implementation of a market environment using a Multi-agent System (MAS), 
where the individual DER units are controlled by local agents that exchange information with a central controller to determine their buying and selling bids.

\section{Tertiary Control}

Tertiary control is the highest level of control and sets long term and typically "optimal" set points depending on the requirements of the host power system. This tertiary control is responsible for coordinating the operation of multiple micro-grids interacting with one another in the system, and communicating needs or requirements from the host grid (voltage support, frequency regulation, etc.). For example, the overall reactive power management of a grid that contains several micro-grids could be accomplished by properly coordinating, through a tertiary control approach, the reactive power injection of generators and micro-grids at the PCC, based on a centralized loss minimization approach for the entire grid. This control level typically operates in the order of several of minutes, providing signals to secondary level controls at micro-grids and other subsystems that form the full grid. Secondary controls, on the other hand, coordinate internal primary controls within the micro-grids and subsystems in the span of a few minutes. Finally, primary controls are designed to operate independently and react in predefined ways instantaneously to local events. Tertiary control can be considered part of the host grid, and not the microgrid itself. Hence, this control level is not discussed further in this paper.

\section{Conclusions}

The development of micro-grids comes as a necessity for the integration of renewable energy sources into remote communities, and as an intermediate milestone towards the realization of the Smart Grid. This paper presented an overview of current developments on micro-grid control, classifying different contributions according to a defined three-level hierarchical structure. The paper reviewed the requirements and desirable traits of the control systems, its different architectures and remaining challenges; emerging approaches applied to micro-grid control (e.g., MPC, MAS) were also discussed. ESS is identified as a key technology for the integration of intermittent renewable energy sources. This, in turn, introduces major challenges to the control system for the appropriate management of this resource. Other challenges for the control of micro-grids include lowinertia of power electronic-interfaced units, low ratio of low voltage grids, uncertainty of generation output and unbalanced system conditions. Some of these challenges have been addressed in the literature, proposing a variety of techniques; however, robustness and adaptiveness remain an issue in most of them.

\section{References}

[1] K. Moslehi and R. Kumar, "A reliability perspective of the smart grid," IEEE Trans. Smart Grid, vol. 1, no. 1, pp. 57-64, Jun. 2010.

[2] R. H. Lasseter, "Microgrids," in Proc. IEEE Power Eng. Soc. Winter Meeting, New York, NY, USA, 2002, pp. 305-308.

[3] R. H. Lasseter and P. Paigi, "Microgrid: A conceptual solution," in Proc. IEEE Power Electron. Spec. Conf., Aachen, Germany, 2004, pp. 4285-4290.

[4] J. Rocabert, A. Luna, F. Blaabjerg, and P. Rodriguez, "Control of power converters in AC microgrids," IEEE Trans. Power Electron., vol. 27, no. 11, pp. 4734-4739, Nov. 2012.

[5] Y. Li, D. M. Vilathgamuwa, and P. C. Loh, "Design, analysis, and real-time testing of a controller for multi-bus microgrid system," IEEE Trans. Power Electron., vol. 19, no. 5, pp. 11951204, Sep. 2004.

[6] J. P. Lopes, N. Hatziargyriou, J. Mutale, P. Djapic, and N. Jenkins, "Integrating distributed generation into electric power systems: A review of drivers, challenges and opportunities," Electric Power Syst. Research, vol. 77, no. 9, pp. 11891203, 2007.

[7] B. Lasseter, "Microgrids [distributed power generation]," in Proc. IEEE Power Engineer. Soc. Winter Meet., Jan. 2001, vol. 1, pp. 146-149.

[8] C. Alvial-Palavicino, N. Garrido-Echeverría, G. Jiménez-Estévez, L. Reyes, and R. Palma-Behnke, "A methodology for community engagement in the introduction of renewable based smart 
microgrid," Energy for Sustainable Development, [13] S. C. Smith, P. K. Sen, and B. Kroposki, vol. 15 , no. 3, pp. 314-323, 2011.

[9] IEEE Standard for Interconnecting Distributed Resources with Electric Power Systems, , 2003, IEEE Std. 1547.

[10] "NETL Modern Grid Strategy—Powering Our 21st-Century Economy: A Compendium of Smart Grid Technologies," White Paper National Energy Technology Laboratory (NETL) for the U.S. Department of Energy Office of Electricity Delivery and Energy Reliability, 2009.

[11] B. Roberts, "Capturing grid power: Performance, purpose, and promise of different storage technologies," IEEE Power Energy Mag., vol. 7, no. 4, pp. 32-41, Jul./Aug. 2009.

[12] P. F. Ribeiro, B. K. Johnson, M. L. Crow, A. Arsoy, and Y. Liu, "Energy storage systems for advanced power applications," Proc. IEEE, vol. 89, no. 12, pp. 1744-1756, Dec. 2001. "Advancement of energy storage devices and applications in electrical power system," in Proc. IEEE Power and Energy Soc. General Meet., Jul. 2008, pp. 1-8.

[14] J. Casazza and F. Delea, Understanding Electric Power Systems: An Overview of Technology, the Marketplace, and Government Regulation, 2nd ed. Hoboken, NJ: John Wiley \& Sons, 2010.

[15] A. F. Burke, "Batteries and ultracapacitors for electric, hybrid, and fuel cell vehicles," Proc. IEEE, vol. 95, no. 4, pp. 806-820, Apr. 2007.

[16] "Master Controller Requirements Specification for Perfect Power Systems (as Outlined in the Galvin Electricity Initiative), "White Paper EPRI and The Galvin Project, Inc., Palo Alto and Chicago, CA and IL, 2007.

[17] A. L. Dimeas and N. D. Hatziargyriou, "Operation of a multiagent system for microgrid control," IEEE Trans. Power Syst., vol. 20, no. 3, pp. 14471455, Aug. 2005 